\title{
Comparison of post-operative pain and quality of life between uniportal subxiphoid and intercostal video-assisted thoracoscopic lobectomy
}

\author{
Jian Chen ${ }^{1}$, Sara Volpi ${ }^{2}$, Jason M. Ali ${ }^{2}$, Giuseppe Aresu ${ }^{2}$, Liang Wu ${ }^{1}$, Zhigang Chen ${ }^{1}$, Jin Wang ${ }^{1}$, \\ Bei Chen ${ }^{1}$, Chenlu Yang ${ }^{1}$, Kostis Marios Soultanis ${ }^{1}$, Gening Jiang ${ }^{1}$, Lei Jiang ${ }^{1}$ \\ ${ }^{1}$ Department of Thoracic surgery, Shanghai Pulmonary Hospital, Tongji University School of Medicine, China; ${ }^{2}$ Department of Cardiothoracic \\ Surgery, The Royal Papworth Hospital, Cambridge, UK \\ Contributions: (I) Conception and design: J Chen, S Volpi, J Volpi, G Jiang, L Jian; (II) Administrative support: KM Soultanis, J Chen, L Jiang; (III) \\ Provision of study materials or patients: J Chen, G Jiang; (IV) Collection and assembly of data: B Chen, Z Chen; (V) Data analysis and interpretation: \\ J Chen, S Volpi, JM Ali, G Aresu, L Jiang; (VI) Manuscript writing: All authors; (VII) Final approval of manuscript: All authors. \\ Correspondence to: Jiang Lei. Department of Thoracic Surgery, Shanghai Pulmonary Hospital, Tongji University, Shanghai 200433, China. \\ Email: jiangleiem@aliyun.com.
}

\begin{abstract}
Background: Uniportal video-assisted thoracoscopic surgery (VATS) although considered less invasive than the multi-port techniques, is still an intercostal approach, resulting in intercostal nerve injury. Recently, some surgeons have tried to address this problem by attempting a subxiphoid approach. The aim of our study was to assess and compare results between intercostal and subxiphoid uniportal VATS lobectomy in terms of postoperative pain and quality of life (QoL).

Methods: Patients from January 2014 to January 2018 undergoing subxiphoid and intercostal VATS lobectomy were prospectively assessed for pain and QoL at 1, 3, and 6 months following discharge. Postoperative pain was measured using a numeric rating scale (NRS) and QoL was assessed with the EuroQoL 5-dimension questionnaire (EQ5D)

Results: Eight hundred and thirty-three patients undergoing lobectomy were included: 373 in the intercostal VATS group and 459 in the subxiphoid group. The proportion of patients with moderate or worse clinical pain was significantly lower at 1 and 3 months after subxiphoid VATS $(\mathrm{P}<0.01)$ compared with intercostal VATS. QoL was significantly higher following subxiphoid VATS at these same time points $(\mathrm{P}<0.001)$.

Conclusions: Uniportal subxiphoid VATS is a safe and feasible minimally invasive approach for undertaking pulmonary lobectomy that may result in reduced postoperative pain compared to conventional VATS. There may also be earlier return of QoL. A randomized controlled trial examining this further would provide further insight into our observations.
\end{abstract}

Keywords: Subxiphoid video assisted thoracoscopic surgery; pain; quality of life (QoL); lobectomy

Submitted Jan 10, 2020. Accepted for publication Jun 02, 2020.

doi: $10.21037 /$ jtd-20-425

View this article at: http://dx.doi.org/10.21037/jtd-20-425

\section{Introduction}

The move towards minimally invasive surgery has been driven by the desire to achieve equivalent surgical outcomes but with reduced morbidity. In thoracic surgery, the evolution from large thoracotomies to video-assisted thoracic surgery has been associated with reductions in postoperative complications, reduced pain, reduced length of hospitalization, earlier return to activities, improved quality of life (QoL), and even possibly survival following lung resection for lung cancer as a result of more patients 
being operable $(1,2)$. Advances in endoscopic imaging systems and instrument design have permitted evolution from initially 3 or 4 port video-assisted thoracoscopic surgery (VATS) techniques to the uniportal technique $(3,4)$. The latter is being increasingly established as the preferred technique as it is the less invasive and, as suggested by some, more ergonomic compared to the other multiport techniques $(5,6)$. However, despite the reduction in the number of ports, uniportal VATS is not without its longterm complications related to the intercostal nerve injury induced by manipulation of the instruments through the intercostal space $(2,7)$.

Pain, a common symptom for which patients seek medical care following thoracic surgery, is an important public health issue that can limit functionality and adversely affect the QoL. Despite VATS being a minimal access technique, studies suggest that up to one-third of patients undergoing VATS procedures develop chronic pain, due to damage or compression of intercostal nerves (7).

Uniportal subxiphoid VATS (SVATS) is an increasingly popular alternative VATS approach which avoids intercostal spaces and therefore prevents damage to the intercostal neurovascular bundles. Uniportal SVATS was initially utilized for non-anatomical lung resections and thymectomy $(8,9)$. However, with experience, the entire range of thoracic surgical procedures have been reported to be performed through SVATS (10-17). We have previously reported data suggesting that SVATS may be associated with reduced post-operative pain following lobectomy compared with uniportal VATS (18). The aim of this study was to compare postoperative pain and QoL at 1, 3 and 6 months postoperatively for patients undergoing lobectomy via SVATS with uniportal VATS. We present the following article in accordance with the STROBE reporting checklist (available at http://dx.doi.org/10.21037/jtd-20-425).

\section{Methods}

\section{Patient population}

This was a prospective comparative study of patients undergoing lobectomy via either the uniportal intercostal or subxiphoid VATS approaches at the Shanghai Pulmonary Hospital. The trial was conducted in accordance with the Declaration of Helsinki (as revised in 2013) and the Harmonized Tripartite Guideline for Good Clinical Practice from the International Conference on Harmonization. The study was approved by the Institutional Review Board of the Shanghai Pulmonary Hospital (IRB No. K17-160). From January 2014 to January 2018, more than 2,100 patients were operated by our group in the Thoracic Surgery Department of the Shanghai Pulmonary Hospital. We diagnosed and staged all patients in accordance with national guidelines (19). The treatment decision was made by a multidisciplinary tumor board. All participants provided written informed consent for participation in this study. Patients were excluded from the study if any of the following criteria were met: (I) previous neoadjuvant therapy including radiotherapy; (II) age $>80$ years to reduce the impact of comorbidity and reduced mobility on QoL assessment; (III) diffuse dense adhesions identified intraoperatively. Clinical characteristics of the patients were obtained from electronic medical records. The study was not randomized and patients underwent SVATS or uniportal intercostal VATS at the surgeons' discretion. Over the period of study there has been a shift in our practice to favoring SVATS, and the period includes our learning curve.

\section{Surgical procedure}

VATS lobectomy is performed using a $10 \mathrm{~mm} 30$-degree thoracoscope. For intercostal lobectomy this was performed via a standard uniportal approach. Subxiphoid VATS was performed as has previously been described (20). A 4-5 cm vertical incision was made in the subxiphoid area and a wound protector was placed. Upon entering the pleural space, the pericardial fat was resected under thoracoscopic guidance. The operation was performed using instruments designed for SVATS (Figure 1). After surgery, a 28F chest tube was positioned through the intercostal incision or inserted through the subxiphoid incision to the pleural apex, and the incision was closed. The unit's standard postoperative management and analgesia protocol was followed for all patients.

\section{Pain and QoL evaluation}

For pain evaluation, we used the 11-point numeric rating scale (NRS) questionnaire, where an NRS score of 0 represented "no pain" and a score of 10 represented "worst pain imaginable". Clinically relevant pain was defined as NRS $\geq 1$. The pain intensity was categorized into two groups: no or mild pain (NRS from 0 to 2 ) and moderate to severe pain (NRS from 3 to 10) (2).

The EuroQoL 5-dimension (EQ5D) questionnaire 

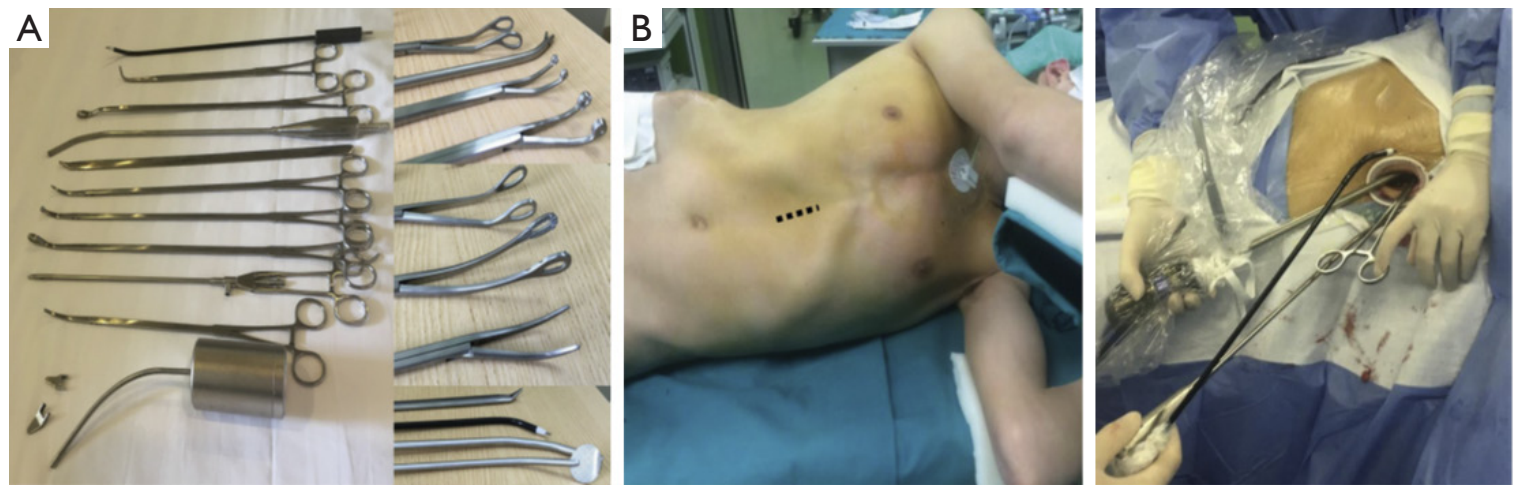

Figure 1 Instruments and positioning for subxiphoid surgery. (A) Instruments designed for subxiphoid video-assisted thoracic surgery (SVATS) (Shanghai Medical Instruments Group) (http://www.jzsf.com/en/index.aspx); (B) intraoperative photos demonstrating the patient positioned in lateral decubitus position with black dashes indicate position of incision and arrangement of instruments through wound protector.

consists of five questions (primary dimensions) referring to mobility, self-care, usual activities, pain-discomfort, and anxiety-depression, which have three mutually excluding answers: "no problems", "some problems", or "extreme problems"). Additionally, EQ5D includes a linear hundred point scale, where 0 is the worst imaginable health state and 100 is the best imaginable health state (21). This instrument is the established tool for assessing QoL at our centre and was chosen due to familiarity and ease of use.

We instructed all patients to answer NRS and the EQ5D questionnaire at 1, 3, 6 months after discharge. These were performed either on attendance at outpatient clinic follow up appointments or by telephone follow-up calls.

\section{Statistical analysis}

We used Student's $t$-test for normally distributed variables to compare means, the Mann-Whitney test to compare non-normally distributed variables, and the $\chi^{2}$ test to compare categorical data. Patients with missing data for a particular variable were excluded from the analysis of that variable. A P value of less than 0.05 was considered statistically significant. All analyses were performed using GraphPad Prism 7.0 (GraphPad Software, San Diego, CA, USA).

\section{Results}

\section{Patient characteristics}

Over the period of study, 832 patients undergoing VATS lobectomy agreed to participate in this study and met inclusion criteria. Of these, 373 (44.8\%) underwent intercostal VATS and 459 (55.2\%) SVATS. Demographic characteristics are summarized in Table 1 . There were no significant differences in terms of age, sex and smoking history. Patients in the subxiphoid group had lower body mass index (BMI) $(23.4 \pm 0.14$ vs. $24.05 \pm 0.18, \mathrm{P}=0.01)$ and superior pulmonary function tests (forced expiratory volume in 1 minute (FEV1): $2.383 \pm 0.028$ vs. $2.247 \pm 0.035, \mathrm{P}=0.002$; FEV1\%: $92.55 \pm 0.772$ vs. $87.07 \pm 0.950, \mathrm{P}<0.001)$. The preoperative albumin and haemoglobin of the two groups were comparable $(\mathrm{P}=0.601$ and $\mathrm{P}=0.549$ respectively). In both groups, right side lobectomies were most commonly, however a higher proportion of those undergoing SVATS were right sided procedures $(\mathrm{P}<0.001)$.

\section{Operative details}

Operative details are summarized in Table 2. The mean operative time was $129.7 \pm 2.2 \mathrm{~min}$ in the subxiphoid group vs. $125.9 \pm 2.2 \mathrm{~min}$ in the intercostal $(\mathrm{P}=0.256)$. The mean number of lymph node stations was equivalent between the two procedures $(\mathrm{P}=0.785)$. The intraoperative blood loss and the first day drainage were similar between the two groups. The postoperative hospital stay was slightly shorter in the subxiphoid group $(4.24 \pm 0.08$ vs. $4.57 \pm 0.09, \mathrm{P}=0.012)$. Perioperative complications were similar between the groups, although there was a slight tendency to increased wound infection rate for patients undergoing intercostal lobectomy $(\mathrm{P}=0.049)$.

\section{Pathology outcomes}

A total of 370 (99.2\%) patients in the intercostal group were diagnosed with lung cancer, 366 (98.1\%). In the subxiphoid group, 451 (98.2\%) cases were lung cancer. Other diagnoses 
Table 1 Demographic characteristics of patients included in the study

\begin{tabular}{|c|c|c|c|}
\hline Variables & Intercostal $(\mathrm{n}=373)$ & Subxiphoid $(n=459)$ & $\mathrm{P}$ \\
\hline Mean & $60.83 \pm 0.54$ & $60.77 \pm 0.43$ & 0.922 \\
\hline$\leq 60$ & $167(44.8)$ & $198(43.1)$ & 0.601 \\
\hline$>60$ & $206(55.2)$ & $261(56.9)$ & 0.082 \\
\hline Male & $196(52.5)$ & $223(48.6)$ & \\
\hline Female & $177(47.5)$ & $236(51.4)$ & \\
\hline Smoking history & & & 0.634 \\
\hline Non-smoker & 272 (72.9) & $342(74.5)$ & \\
\hline FEV1 & $2.247 \pm 0.035$ & $2.383 \pm 0.028$ & 0.002 \\
\hline FEV1\% & $87.07 \pm 0.950$ & $92.55 \pm 0.772$ & $<0.001$ \\
\hline Preoperative albumin (mmol/L) & $39.24 \pm 0.26$ & $39.37 \pm 0.26$ & 0.601 \\
\hline Preoperative haemoglobin (g/dL) & $131 \pm 0.72$ & $130 \pm 0.76$ & 0.549 \\
\hline \multicolumn{4}{|l|}{ TNM staging (7th Edition) } \\
\hline $1 \mathrm{a}$ & $150(40.2)$ & $298(64.92)$ & $<0.001$ \\
\hline $1 b$ & $126(33.8)$ & $91(19.83)$ & \\
\hline $2 a$ & $40(10.7)$ & $30(6.54)$ & \\
\hline Squamous cell carcinoma & 16.50 & 19.9 & \\
\hline Other & 5.80 & 4.20 & \\
\hline
\end{tabular}

Data present as mean \pm SD or percentage. BMI, body mass index; FEV1, forced expiratory volume in 1 minute.

included granuloma, tuberculosis, bronchiectasis, cyst and hamartoma. The staging, according to the $7^{\text {th }}$ edition The American Joint Committee on Cancer (AJCC)/Union for International Cancer Control (UICC) tumour, node, metastasis (TNM) staging system is presented in Table 1. There was a significantly greater proportion of patients in the subxiphoid group with stage 1a tumours. All tumours were fully resected and all resections were reported $\mathrm{R}_{0}$.

\section{Patient pain score and quality of life assessment}

The pain questionnaires and EQ5D scores were completed by $334(89.5 \%)$ patients in the intercostal and 309 (67.3\%) patients in the subxiphoid group. The answers revealed a higher pain score in the intercostal group at 1 and 3 months $(\mathrm{P}=0.002$ and $\mathrm{P}=0.011$ respectively) (Figure 2$)$.

EQ5D scores were significantly higher in the subxiphoid group compared with the intercostal group at all three 
Table 2 Postoperative details and outcomes

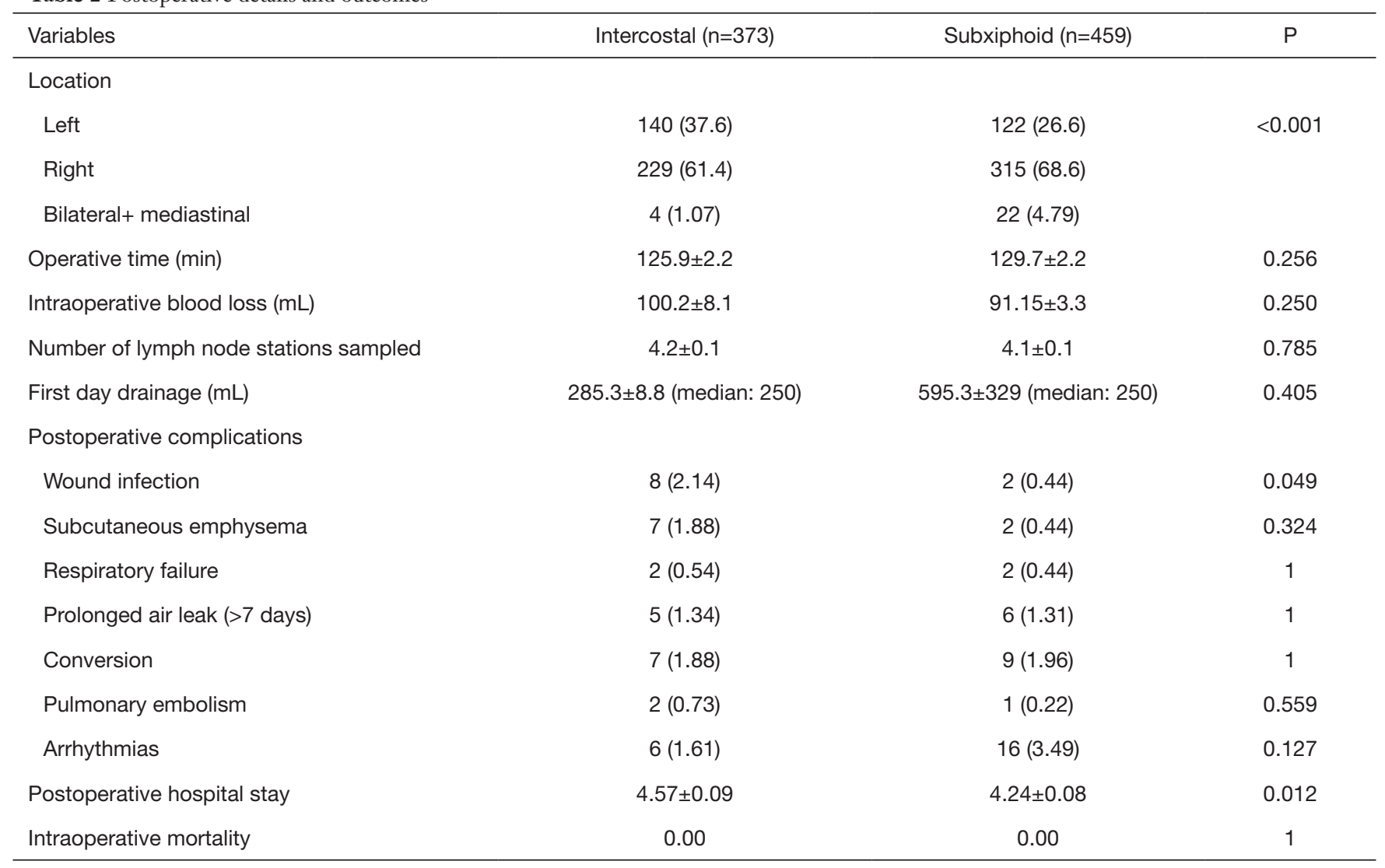

Data present as mean \pm SD or percentage.

assessment times: 1,3 , and 6 months postoperatively $(\mathrm{P}<0.001, \mathrm{P}<0.001$ and $\mathrm{P}=0.003$, Figure 3).

\section{Discussion}

In this study we aimed to compare post-operative pain and quality of life of patients undergoing VATS lobectomy via the uniportal intercostal route with the subxiphoid route. We demonstrate that patients undergoing SVATS experienced less pain and experienced a greater quality of life during the early post-operative period, supporting the belief that disruption of the intercostal neurovascular bundles is an important determinant of pain following thoracic surgery, and by avoiding the intercostal spaces, SVATS offers an alternative approach that is associated with less post-operative pain.

Although existing data suggest no differences regarding mortality and morbidity, length of stay, number of dissected lymph nodes and survival between uniportal and conventional three port VATS, there is a trend towards shifting to the uniportal technique, probably due to it being even less invasive and more ergonomic (4,22-25). Interestingly, while both exhibit a clear advantage over thoracotomy in the aforementioned fields, accumulating data indicate that almost $30 \%$ of patients undergoing a VATS procedure still experience clinically significant pain, due to intercostal nerve compression or injury (7). This has brought forward the assumption that the subxiphoid approach could reduce the incidence of postoperative pain and chest-wall paresthesia, since there are not intercostal nerves running along the dissection plane of the subxiphoid area.

Since its first implementation in 2012, the uniportal subxiphoid approach has been used for thymomas, metastasectomy, hyperhidrosis, spontaneous pneumothorax, and anatomical lung resections_-including pneumonectomies $(9,12,20,26,27)$. SVATS can be challenging to learn and challenges early in the learning curve include difficulty controlling a bleeding incidence, due to the distance between the subxiphoid incision and the site of the vascular 


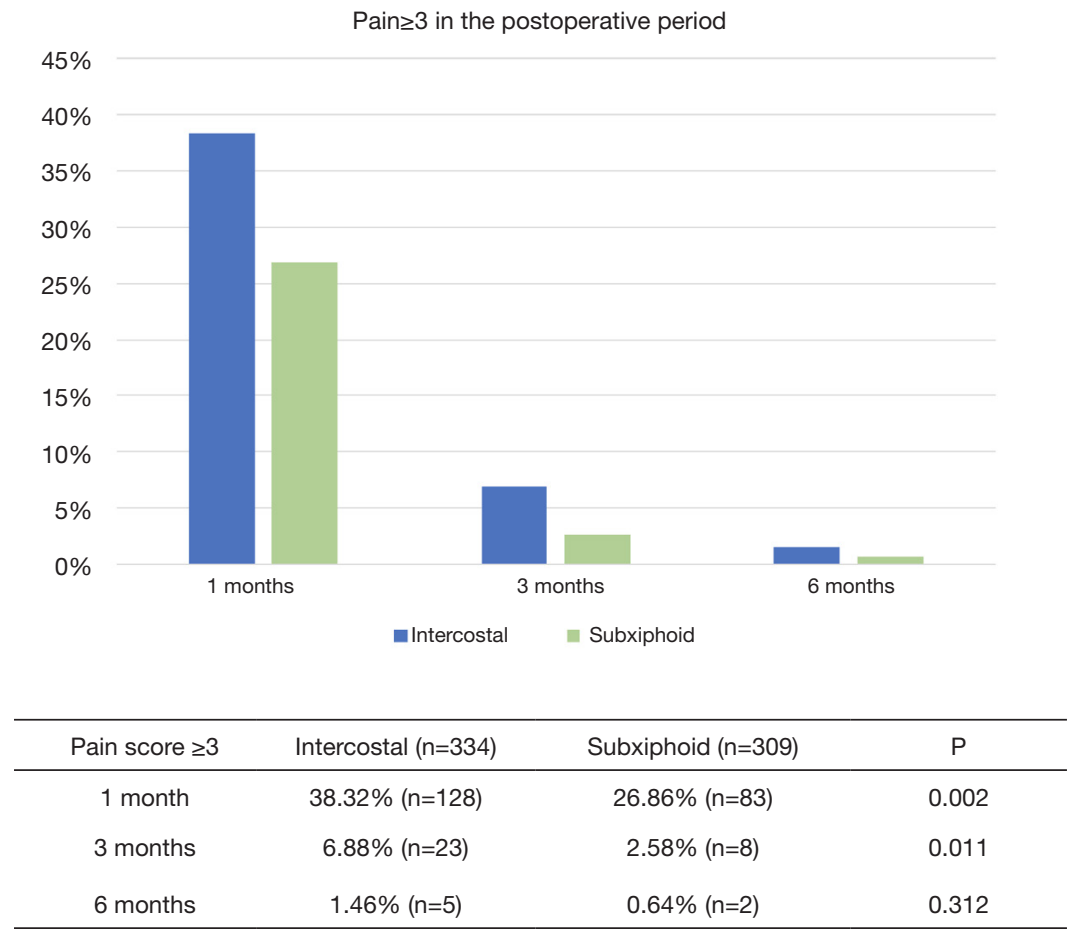

Figure 2 Moderate to severe pain rate at 1, 3 and 6 months postoperatively.

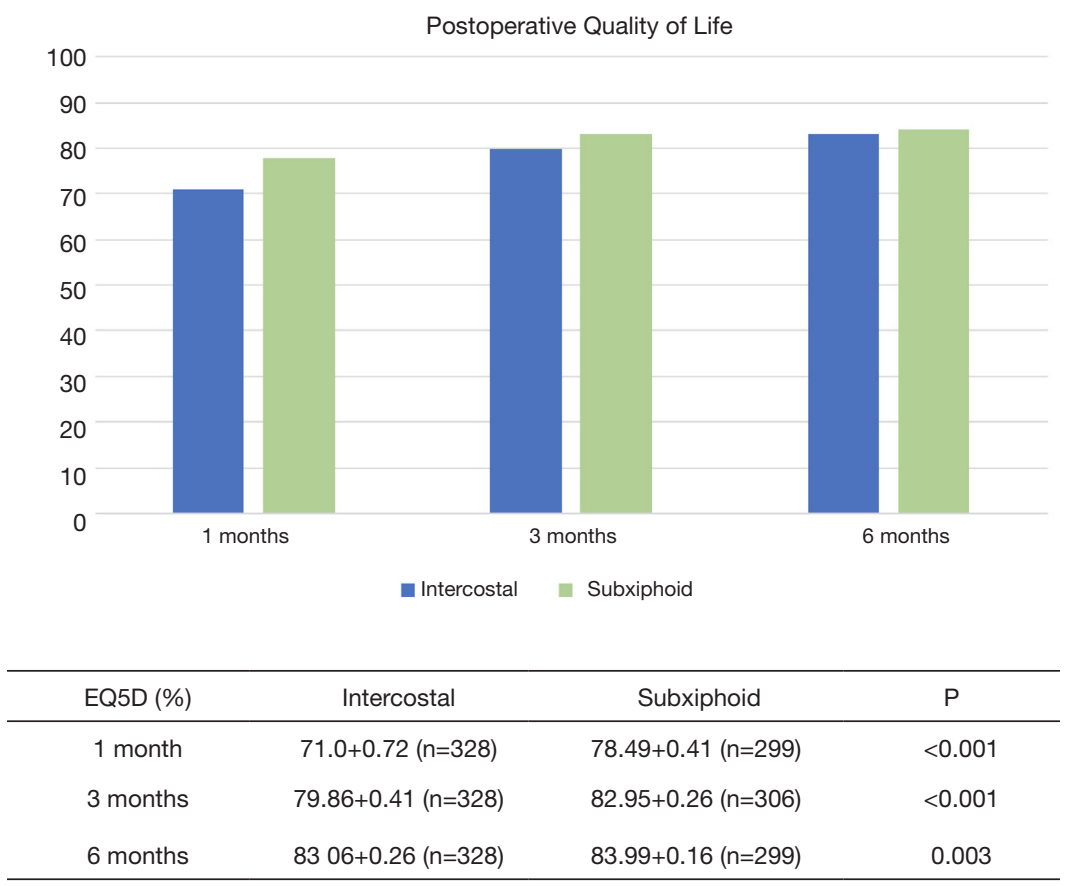

Figure 3 Quality of life at 1, 3 and 6 months postoperatively. 
injury and difficulty conducting a complete mediastinal lymphadenectomy. However, with experience these are not hurdles to using this approach. This study includes patients during our learning curve for SVATS as such there was some selection early in the period for patients we perceived to be easier SVATs candidates. It is important to acknowledge that SVATS is more challenging in left sided procedures due to the heart's location, and this is reflected in the significantly higher proportion of right sided procedures in the SVATS group. Furthermore, this approach can be challenging in obese patients, and we observe a lower mean BMI in the SVATS group in keeping with this.

Regarding postoperative pain, it is believed that injury to the intercostal nerve induced by the maneuvering of the instruments through the intercostal space is an important etiological factor (28). The post-operative pain experienced by the patients undergoing SVATS was significantly lower at all three time points suggesting that avoidance of intercostal nerve handling and injury may lead to reduced short and medium-term pain following VATS procedures. The average pain scores at 1, 3, 6 after subxiphoid surgery were significantly lower compared to those of the intercostal group $(\mathrm{P}<0.001)$, suggesting that avoiding the intercostal neurovascular bundle may be a way to reduce postoperative intercostal neuralgia which can leads to chronic pain and paresthesia. Furthermore, more patients in the intercostal group reported moderate-to-severe pain (NRS $\geq 3$ ) than did those in the SVATS group.

QoL after surgery has received increasing attention over the past decade as a more important outcome than survival alone $(29,30)$. The use of different questionnaires to assess the QoL makes comparisons between different studies difficult but results from one randomized study that compared VATS lobectomy with thoracotomy demonstrated improved QoL in patients undergoing VATS (2). In our present study, QoL, as assessed by EQ5D, was significantly higher in patients undergoing SVATS compared with intercostal uniportal VATS. This in part may be attributed to the reduced postoperative pain experienced by the patients in the subxiphoid group.

There are limitations of our study. Firstly, there was no randomization in this work to SVATS $v s$. intercostal VATS and so there is the potential for bias that may have impacted on our findings. This is important, since there were some differences between the patient groups-for example, generally a lower TNM stage. The aim of this retrospective analysis was to investigate the presence of a trend to inform a more comprehensive analysis in the shape of a randomized controlled trial. Secondly, almost $30 \%$ of the patients were lost during follow up and did not complete any or all of the questionnaires at the three time points. We don't know if the results would have been different should we have had a higher completion rate. The operations in this study were all performed by one group in a single center with already established expertise in uniportal VATS. Certainly, prospective studies, especially if comparative and randomized could contribute a lot towards defining the pros and cons, the indications and contraindications of the two techniques. There are more comprehensive tools to assess QoL, but we chose to use the tool that was established and for which there was already familiarity for the staff involved in data collection. It is important to acknowledge that assessment of pain and QoL is subjective.

In conclusion, uniportal subxiphoid VATS is a safe and feasible minimally invasive approach for undertaking pulmonary lobectomy that may result in reduced postoperative pain compared to conventional VATS. There may also be earlier return of quality of life. A randomized controlled trial examining this further would provide further insight into our observations.

\section{Acknowledgments}

Funding: This study was supported by the Fundamental Research Funds for the Central Universities (22120180019) and Health and Family Planning Commission of Shanghai Municipality (20184Y0090).

\section{Footnote}

Reporting Checklist: The authors have completed the STROBE reporting checklist. Available at http://dx.doi. org/10.21037/jtd-20-425

Data Sharing Statement: Available at http://dx.doi. org/10.21037/jtd-20-425

Conflicts of Interest: All authors have completed the ICMJE uniform disclosure form (available at http://dx.doi. org/10.21037/jtd-20-425). JM Ali serves as an unpaid editorial board member of Fournal of Thoracic Disease from Oct 2019 to Sep 2021. The other authors have no conflicts of interest to declare.

Ethical Statement: The authors are accountable for all aspects of the work in ensuring that questions related 
to the accuracy or integrity of any part of the work are appropriately investigated and resolved. The trial was conducted in accordance with the Declaration of Helsinki (as revised in 2013) and the Harmonized Tripartite Guideline for Good Clinical Practice from the International Conference on Harmonization. The study was approved by the Institutional Review Board of the Shanghai Pulmonary Hospital (IRB No. K17-160). All patients enrolled completed the informed consent form.

Open Access Statement: This is an Open Access article distributed in accordance with the Creative Commons Attribution-NonCommercial-NoDerivs 4.0 International License (CC BY-NC-ND 4.0), which permits the noncommercial replication and distribution of the article with the strict proviso that no changes or edits are made and the original work is properly cited (including links to both the formal publication through the relevant DOI and the license). See: https://creativecommons.org/licenses/by-nc-nd/4.0/.

\section{References}

1. Improving rates of surgery for lung cancer. Lancet 2011;378:1678.

2. Bendixen M, Jørgensen OD, Kronborg C, et al. Postoperative pain and quality of life after lobectomy via video-assisted thoracoscopic surgery or anterolateral thoracotomy for early stage lung cancer: a randomised controlled trial. Lancet Oncol 2016;17:836-44.

3. Boffa DJ, Kosinski AS, Furnary AP, et al. Minimally Invasive Lung Cancer Surgery Performed by Thoracic Surgeons as Effective as Thoracotomy. J Clin Oncol 2018;36:2378-85.

4. Wang L, Liu D, Lu J, et al. The feasibility and advantage of uniportal video-assisted thoracoscopic surgery (VATS) in pulmonary lobectomy. BMC Cancer 2017;17:75.

5. Rocco G. One-port (uniportal) video-assisted thoracic surgical resections--a clear advance. J Thorac Cardiovasc Surg 2012;144:S27-31.

6. Migliore M. Initial History of Uniportal Video-Assisted Thoracoscopic Surgery. Ann Thorac Surg 2016;101:412-3.

7. Bayman EO, Parekh KR, Keech J, et al. A Prospective Study of Chronic Pain after Thoracic Surgery. Anesthesiology 2017;126:938-51.

8. Suda T, Sugimura H, Tochii D, et al. Single-port thymectomy through an infrasternal approach. Ann Thorac Surg 2012;93:334-6.

9. Suda T, Ashikari S, Tochii S, et al. Single-incision subxiphoid approach for bilateral metastasectomy. Ann Thorac Surg 2014;97:718-9.

10. Hernandez-Arenas LA, Lin L, Yang Y, et al. Initial experience in uniportal subxiphoid video-assisted thoracoscopic surgery for major lung resections. Eur J Cardiothorac Surg 2016;50:1060-6.

11. Ali JM, Kaul P, Jiang L, et al. Subxiphoid pneumonectomy: the new frontier? J Thorac Dis 2018;10:4464-71.

12. Aresu G, Jiang L, Bertolaccini L. Subxiphoid videoassisted major lung resections: the Believers' speech. J Thorac Dis 2017;9:E387-9.

13. Aresu G, Wu L, Lin L, et al. The Shanghai Pulmonary Hospital subxiphoid approach for lobectomies. J Vis Surg 2016;2:135.

14. Guido Guerrero W, Hernandez Arenas LA, Jiang G, et al. Subxiphoid mediastinal lymphadenectomy. J Vis Surg 2016;2:105.

15. Hernandez-Arenas LA, Lin L, Wu L, et al. Subxiphoid uniportal video-assisted thoracoscopic trisegmentectomy. J Vis Surg 2016;2:90.

16. Weaver H, Ali JM, Jiang L, et al. Uniportal subxiphoid video-assisted thoracoscopic approach for thymectomy: a case series. J Vis Surg 2017;3:169.

17. Aresu G, Weaver H, Wu L, et al. Uniportal subxiphoid video-assisted thoracoscopic bilateral segmentectomy for synchronous bilateral lung adenocarcinomas. J Vis Surg 2016;2:170.

18. Song N, Zhao DP, Jiang L, et al. Subxiphoid uniportal video-assisted thoracoscopic surgery (VATS) for lobectomy: a report of 105 cases. J Thorac Dis 2016;8:S251-7.

19. Ettinger DS, Aisner DL, Wood DE, et al. NCCN Guidelines Insights: Non-Small Cell Lung Cancer, Version 5.2018. J Natl Compr Canc Netw 2018;16:80721.

20. Ali J, Haiyang F, Aresu G, et al. Uniportal Subxiphoid Video-Assisted Thoracoscopic Anatomical Segmentectomy: Technique and Results. Ann Thorac Surg 2018;106:1519-24.

21. Szende A, Janssen B, Cabases J. editors. Self-Reported Population Health: An International Perspective based on EQ-5D. Dordrecht: Springer, 2014.

22. Bertolaccini L, Viti A, Terzi A, et al. Geometric and ergonomic characteristics of the uniportal video-assisted thoracoscopic surgery (VATS) approach. Ann Cardiothorac Surg 2016;5:118-22.

23. Abu Akar F, Gonzalez-Rivas D, Ismail M, et al. Uniportal video-assisted thoracic surgery: the Middle East 
experience. J Thorac Dis 2017;9:871-7.

24. Surendrakumar V, Martin-Ucar AE, Edwards JG, et al. Evaluation of surgical approaches to anatomical segmentectomies: the transition to minimal invasive surgery improves hospital outcomes. J Thorac Dis 2017;9:3896-902.

25. Kutluk AC, Kocaturk CI, Akin H, et al. Which is the Best Minimal Invasive Approach for the Treatment of Spontaneous Pneumothorax? Uniport, Two, or Three Ports: A Prospective Randomized Trail. Thorac Cardiovasc Surg 2018;66:589-94.

26. Li L, Tian H, Yue W, et al. Subxiphoid vs intercostal single-incision video-assisted thoracoscopic surgery for spontaneous pneumothorax: A randomised controlled trial. Int J Surg 2016;30:99-103.

27. Fok M, Karunanantham J, Ali JM, et al. Subxiphoid approach for spontaneous bilateral pneumothorax: a case report. J Vis Surg 2017;3:146.

28. Siu E, Quick JS, Xu X, et al. Evaluation of the Determinants of Satisfaction With Postoperative Pain Control After Thoracoscopic Surgery: A Single-Center, Survey-Based Study. Anesth Analg 2019;128:555-62.

29. Park BJ, Ishill NM, Flores RM, et al. Prospective comparison of postoperative quality of life (QOL) in patients undergoing lobectomy by video-assisted thoracic surgery (VATS) versus thoracotomy for non-small cell lung cancer (NSCLC). J Clin Oncol 2008;26:7543.

30. Fernando HC, Landreneau RJ, Mandrekar SJ, et al. Analysis of longitudinal quality-of-life data in highrisk operable patients with lung cancer: results from the ACOSOG Z4032 (Alliance) multicenter randomized trial. J Thorac Cardiovasc Surg 2015;149:718-26.
Cite this article as: Chen J, Volpi S, Ali JM, Aresu G, Wu L, Chen Z, Wang J, Chen B, Yang C, Soultanis KM, Jiang G, Jiang L. Comparison of post-operative pain and quality of life between uniportal subxiphoid and intercostal video-assisted thoracoscopic lobectomy. J Thorac Dis 2020;12(7):3582-3590. doi: 10.21037/jtd-20-425 